\title{
Experimental study on combined defrosting performance of heat pump air conditioning system for pure electric vehicle in low temperature
}

Guanghui Zhou ${ }^{1}$, Haijun $\mathrm{Li}^{1 *}$, Enhai $\mathrm{Liu}^{1}$, Bo Li ${ }^{2}$, Yuying $\mathrm{Yan}^{2}$, Tong $\mathrm{Chen}^{3}$, Xiaonan Chen ${ }^{1}$

${ }^{1}$ School of Energy \& Environmental, Zhongyuan University of Technology, Zhengzhou,450007, China

${ }^{2}$ Fluids \& Thermal Engineering Research Group, Faculty of Engineering, University of Nottingham, UK

${ }^{3}$ China Aviation Lithium Battery CO., LTD., Luoyang, 471000, China

Abstract: The development of defrosting technology is a crucial technical barrier to the application of_the heat pump air conditioning system for the pure electric vehicle. The frosting on the air conditioning system significantly affects systematic performance and reliable operation especially in low temperature and high humidity climate condition. Therefore, in this paper, an experimental study of low low-temperature heat pump air conditioning system with the combined defrost technology of increasing enthalpy and temperature is carried out in order to find proper thermal management solutions. Based on the reverse-cycle methods, the combined defrost technology makes full use of the compressor air-supplying enthalpy-adding, air-cooled heat exchanger inside the vehicle preheating, temperature-raising, enthalpy-adding and the external heat exchanger condensation temperature-increasing technologies. The fast defrosting process can be realized by means of releasing the condensation heat and volume significantly while the external outer heat exchanger is conducting a defrosting operation. Meanwhile, the cold cabin eold-sensitivity can be reduced eorrespondingly while defrosting process taking place correspondingly. Experimental results shows that under the operating condition of $-20^{\circ} \mathrm{C}$ outside environment temperature and $80 \%$ relative humidity, rapid-instant defrosting time at fully defrosted aircooled heat exchanger outside the vehicle can be controlled within 100 seconds.

Keywords: Heat pump, Pure electric vehicle, Air conditioning, Defrosting, Thermal management

\section{Introduction}

With the intensifying energy crisis and the increasing environmental problems, it is imperative to take actions for energy saving and environmental protections on automotive industries. Pure electric vehicle (PEV) as disruptive technology provide an alternative way to replace traditional automotive industry-in-order to achieve sustainable development in near futureshortly. To maintain the PEV working at proper temperature and humidity condition, vehicular air conditioning system becomes an indispensable sub-system that it not only provides the thermal comfort in the cabin but also contributes to the safeties of traction batteries and power electroniesfelectronics [1,2]. The wide-ranging features of the eonstantly ehangingevolving environment outside the car has also raised the requests on the air conditioning system performance in the PEV. And the heat pump air conditioning system has such advantages as high efficiency, energy saving, environmental protection that it is becoming the priority of vehicular air conditioning in the PEV[3-6]. There are, in spite of 
42 aforementioned-merits mentioned above, still some issues of itself especially in low temperature and high humidity ambient. The exterior heat exchanger of the heat pump prone to frost while the system is in heating mode. It not only causes both blockages of air channels and increase of ventilation resistance, but also the overall thermal resistance of exterior heat exchanger. Consequently, the frost will be accumulated and thickened resulting in serious severe deterioration of working performance and reliability of vehicular conditioning system[system [7-9]. Therefore, it is important to find out the main factors of influencing defrost in the exterior heat exchanger-in order-to reduce defrosting time through reasonable and effective control strategies.

An eEvaporator is a unit where it is easy to frost, so to analyze operating characteristics of the evaporator in the case of defrosting is the key to studying the defrosting of the heat pump system. In the 1970s, Sanders, a Dutch[10] began the study on defrosting of air-conditioning evaporators, who created a model of evaporator defrosting of the air-conditioning system in his doctoral thesis, and recorded the whole course of defrosting through experiments and analyzed the distribution of energy consumption in the system during the defrosting. In the 1980s, Е.N.АНДРАЧНИКОВ, a scholar of the former Soviet Union[11] proposed an effective efficient way of automatic evaporator defrosting, and the system was designed with main and secondary evaporators, and a 2_PM time relay was used for control over the two evaporators defrosting alternately. D. L O'Neal and Payne[12] studied the effect of the air volume of the evaporator fan on the defrosting performance. Based on the basic air volume of $72 \mathrm{~m}^{3} / \mathrm{min}$, experiments were conducted at a low air volume of $40 \mathrm{~m}^{3} / \mathrm{min}$ and a high air volume of $88 \mathrm{~m}^{3} / \mathrm{min}$ respectively. The results showed that compared with the basic air volume, the defrosting time and water accumulation after defrosting were greatly

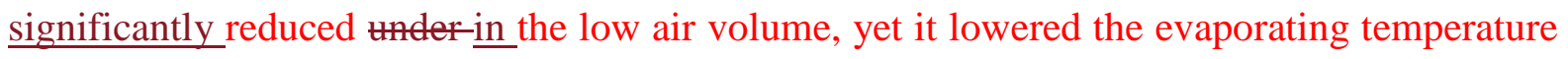
of the system and increased the frosting rate of heat exchanger; under the high air volume, the heat exchange of the system was increased, meanwhile, the defrosting time was prolonged, yet it increased the evaporating temperature of the system which lowered the frosting rate of the heat exchanger. Padhmanabhan[13] compared the performance difference between finned evaporator and micro-channel evaporator during defrosting, and found that the defrosting time of the finned evaporator was about twice of that of micro-channel evaporator, but the frosting rate of the micro-channel was ebviously apparently higher than that of the finned evaporator.

In recent years, computers are used to simulate and analyze the defrosting performance of the heat pump system, which has conservation equation, created a hot air defrosting dynamic cycle dynamic-model, aiming to simulate the performances of evaporator and condenser at reverse cycle defrosting,-besides, tThrough experiments, the model proved that it could not only simulate characteristics of defrosting of the system, but also simulate-affect the whole defrosting course. Dopazo[15] created a heat pump evaporator defrosting model on the basis of hot air, the model divided the defrosting process into six stages: preheating, defrosting outside tubes, defrosting of fins, induced air, water film formed on fins surfaces, drying and heating, the control volume in each stage was represented by a node in the system model. A finite difference method is used to solve the equation, and the results included time needed for defrosting, energy distribution 
during defrosting, characteristics of instantaneous refrigerant and temperature distribution of finned tubes. $\mathrm{Qu}[16,17]$ firstly studied characteristics-features of the multi-tube heat exchanger in defrosting, and the results showed that the defrosting time of upper layers of tubes was faster than that of the lower ones, the defrosting efficiency was estimated to be $34.5 \%$. In order to quantitatively analyze the effect of different-various layers of tubes on defrosting, he created a semi-empirical mathematical model and the defrosting time trend calculated for different layers from top to bottom was the same as the conclusion of the experiment, and pointed out that the frosting time of lower layers of tubes reduced the defrosting efficiency of the system.

At present, it is still at the preliminary stage that there are few researches on defrosting of heat pump PEV air conditioning system. Zhong Hua and others make defrosting control in terms ofregarding traditional vehicle design in combination with the electronic expansion valve, and enhance air volume of the evaporator while increasing the electronic expansion valve epeningfopening [18]. Wu et al. find out through experiment that while the heat pump air conditioning system for PEV is supplying heat atin low temperature, the outdoor micro channel heat exchanger was frosted severely, which influences the heating capacity of the system and the coefficient of performance, but the defrosting solution is not proposed[19]. Therefore, the experimental temporary tables of_low-low-temperature heat pump air conditioning system for PEV is designed[9], and the condensation temperature and defrosting speed under different working conditions are tested. In addition, the variants are analysed such as system cooling capacity, exhaust temperature, outlet air temperature and the import and export temperature of exterior heat exchanger along with the changes of system defrost operating time. The influence on exterior heat exchanger defrosting performance by different factors isare also studied in order to determine the fast and reliable defrosting method-and provide an experimental basis for further improvement of the performance of PEV air conditioning system.

\section{Heat pump type air conditioning system for PEV}

The test rig of a low-temperature heat pump air conditioning system for PEV is designed and established using the quasi-two-stages compression principle. It combines with both the characteristics of low--temperature heat pump technology and automotive air conditioning conditions as shown in Fig.1. This test rig consists of a compressor, four-way valve, aircooled heat exchanger outside the vehicle, one-way valve, liquid storage drier, main expansion valve, air-cooled heat exchanger inside the vehicle, air-supplying expansion valve, and intermediate heat exchanger as well as other auxiliary parts.

The system can achieve multiple basic working modes of electric vehicle cooling, battery electric heating, and air-cooled heat exchanger outside the vehicle defrosting under different working conditions. In cooling mode, the four-way valve switches into the cooling channel which is the same as erdinary conventional car air conditioning cooling processes. The circulating refrigerant is discharged through compressor with high pressure and subsequently flow into air-cooled heat exchanger outside the vehicle for condensation process. Thereafter After that, it flows into liquid storage drier through the one-way valve. Then the refrigerant 
127 flows into the main expansion valve after going through the intermediate heat exchanger, and 128 then low pressure and low_-temperature flow enters into an air-cooled heat exchanger inside 129 the vehicle for evaporation through the one-way valve. Eventually, it is absorbed by the 130 compressor after going through the four-way valve.

131 In heating mode, the four-way valve switches into the heating channel, the circulating 132 refrigerant is discharged throughfrom the compressor at high pressure and high--temperature state. Then the flow enters into an air-cooled heat exchanger inside the vehicle for condensation purpose, later on ${ }_{2}$ it flows into the liquid storage drier through the one-way valve and directly flows into the main circulating pipeline of the intermediate heat exchanger. Different from the cooling mode, the circulating refrigerant isare separated into two streams 137 after flowing out of the intermediate heat exchanger in order to achieve both mass flow and 138 temperature reduction by the air-supplying expansion valve. The auxiliary circulating 139 pipeline isare formed in order to exchange heat in the intermediate heat exchanger with 140 circulating refrigerant in the main circulating pipeline. This- $\underline{\text { It }}$ will help increase the degree of the pipeline. On the other hand, the circulating refrigerant in main-primary circulating pipeline flows into the air-cooled heat exchanger outside the vehicle for evaporation purpose through the one-way valve after mass flow and temperature reduction in the air-supplying expansion valve and main expansion valve. Finally, the main flow isare absorbed by the compressor through the four-way valve, and it is also blended with the circulating refrigerant from an auxiliary pipeline in the compression chamber in which the flow are from airsupplying opening through the one-way valve.

148 When the air-cooled heat exchanger outside the vehicle is in defrosting mode, the reverse cycle rapid defrost technology of enthalpy and temperature rise can be applied. The four-way valve switches into cooling mode, the circulating refrigerant are discharged by the compressor and then flow into the air-cooled heat exchanger outside the vehicle through the four-way valve for condensation and defrosting purposes. Afterwards, the refrigerants flows into the liquid storage drier through the one-way valve, and high--pressure refrigerants flow into the main expansion valve through the intermediate heat exchanger. Eventually, the flow with low pressure and low_-temperature enters into_an air-cooled heat exchanger inside the vehicle for evaporation process and the full cycle will be finished by the compressor through the same four-way valve.

The theoretical cycle of the electric vehicle-based low-temperature heat pump airconditioning system, designed by this article, is shown in Fig.2, the working medium in air recirculation pipelines enters the compressor suction cavity of compressor through the intake port on the compressor ender cover, which is an insulating and throttling process[20]: After the working medium in the recirculation pipeline travels through the intake port, a middle pressure is reduced to be the suction pressure of compressor, there are a few circulating medium mixed with the suction of the compressor in the recirculation pipelines. During the course, the superheat of circulating medium was reduced in the compression cavity of the compressor, after compression, the discharge temperature was reduced, or there are still a few liquid of circulating medium, according to the insensitiveness of scroll compressor to wet 
generated due to vapour compression and friction of kinetic and stationary scroll plates of compressor during the compression, which greatly reduced the air discharge temperature of the compressor, as shown in Fig. 2, the process from 9' to 2'. The mixture of working medium

172 in the recirculation pipelines and that of compressor suction cycle reduce the superheat of working medium in the compression cavity from the compressor or some carry a little liquid, which both increase the quality and flow of working medium for compression in the compressor. Thus it increased-improved the quality and flow of the working medium in the condensing process and increased the heating capacity of the heat pump system [21]. The working medium in the recirculating pipelines will evaporate and absorb heat in the middleintermediate heat exchanger-, which is a latent heat ofin vaporization, yet the liquid working medium in the condensing heat pump in another pipeline of the middleintermediate 180 heat exchanger released heat, which is a sensible heat. The liquid medium ork the heat 181 pump before entering the main expansion valve has a great super-cooling effect, which 182 increases the cooling capacity of the heat pump working medium in the evaporator. While, 183 the super-cooling is limited by the mixed air pressure, that is, the working medium of the 184 lowest main loop heat pump is super-cooling which exceeds the saturated temperature 185 corresponding to the air supply pressure. After working medium in the air recirculation 186 pipelines is mixed with that of the air intake heat pump of the compressor, it will increase the 187 quality and flow of working medium of the heat pump in the compression cavity of the 188 compressor, so the compressor power is increased a little. Meanwhile, the middleintermediate 189 heat exchanger increases the super-cooling of heat pump working medium in the main 190 expansion valve, the refrigerating capacity of the heat pump system is increased in the 191 evaporator. The expansion valve of recirculation controls the air pressure and the quality and 192 flow of working medium in the pipelines, if losing the air expansion valve, the air 193 recirculation pressure and the quality and flow of working medium will rise; vice versa it will 194 drop, which has higher influence to the performance of air circulation-based heat pump air 195 conditioning system.

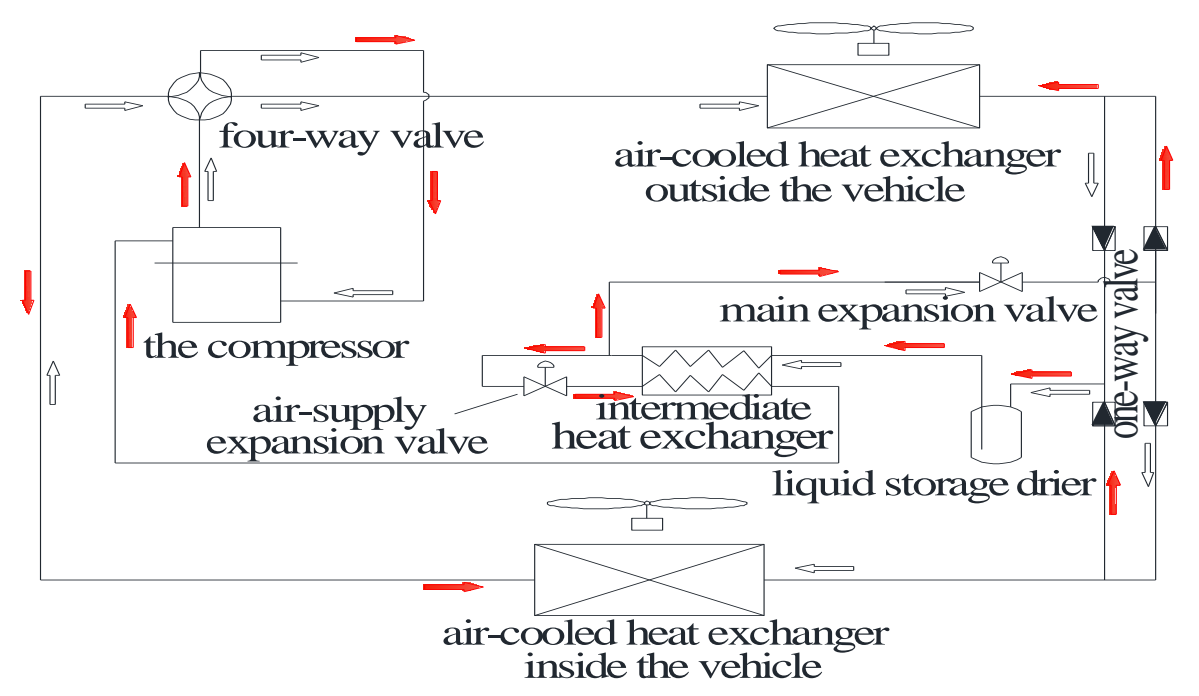

the heating mode

the refrigeration mode

Fig.1. Diagram of the heat pump air conditioning system of the pure electric vehicle. 


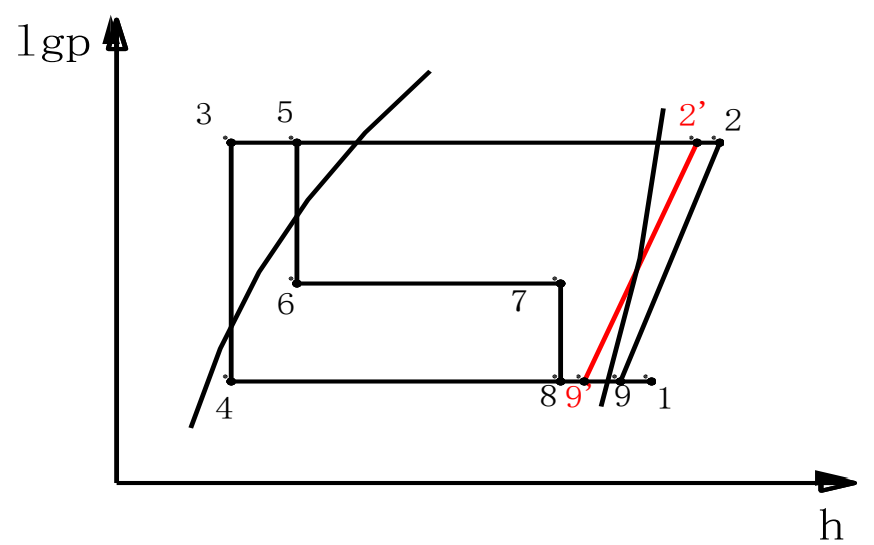

Fig.2. Log p-h diagram of the heat pump cycle with middle heat exchanger

\section{3. The Modelling of Concentric Cylindrical Thermoelectric Generator}

197 The experiment is to study the characteristics of the defrosting mode in heat pump air 198 conditioning system of $\mathrm{PEV}_{\llcorner}$, therefore -Therefore heating is supplied within the cabin ${ }_{2}$ and 199 the air conditioning system is working at low temperature and high humidity condition which 200 leads to heat exchanger frosting outside the vehicle. The frosting conditions of this 201 experiment are described in Table1.

Table 1 The working condition of frosting for the experiment.

\begin{tabular}{cc}
\hline Working condition & Parameters \\
\hline Ambient temperature outside the vehicle $/{ }^{\circ} \mathrm{C}$ & -20 \\
Ambient temperature inside the vehicle $/{ }^{\circ} \mathrm{C}$ & $15 、 20 、 25$ \\
Relative air humidity outside the vehicle $/ \%$ & 80 \\
Revolving speed of compressor $/ \mathrm{rpm}$ & 5000 \\
Air output of fan inside the vehicle $/\left(\mathrm{m}^{3} / \mathrm{h}\right)$ & 540 \\
Fan speed outside the vehicle $/(\mathrm{m} / \mathrm{s})$ & 4.5 \\
Air supplement rate $/ \%$ & 30 \\
\hline
\end{tabular}

204 If the wind speed at air-cooled heat exchanger outside the vehicle is lower than $0.2 \mathrm{~m} / \mathrm{s}$, it 205 is noted that the frosting of air-cooled heat exchanger outside the vehicle completes 206 thoroughly. The frosting scenario is shown inas Fig. 3. 


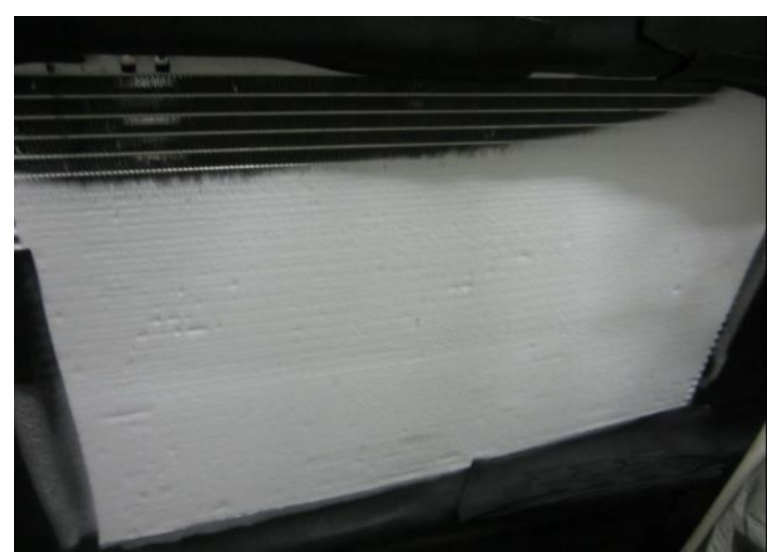

Fig. 3. Complete frosting of heat exchanger outside the vehicle.

207 In the present study, the composite defrosting technology is adopted based on the 208 defrosting of reversed cycle, integrally uses the technology of increasing enthalpy by air209 supplying of compressor, technology of preheating and raising temperature and increasing 210 enthalpy of heat exchanger inside the vehicle, technology of increasing condensing 211 temperature of heat exchanger outside the vehicle, to make heat release of condensation and 212 heat transfer temperature difference of condensation increase obviously, which realize 213 defrosting rapidly and reduce draft of cold air supply inside the vehicle when defrosting. Fig. 2144 shows the defrosting of heat exchanger outside the vehicle, which is taken after the 215 defrosting begins with an interval of 20 seconds. Fig. 4(a) is taken when defrosting begins. 216 Fig. 4(b) is after defrosting operates 20 seconds. At that time, the air exhaust of compressor 217 passes upside of heat exchanger outside the vehicle to melt the frost here firstly. Fig. 4(c) is 218 after defrosting operates 40 seconds. At that moment, the defrosting area of heat exchanger 219 outside the vehicle is enlarged. After defrosting eperates-runs 60 seconds (Fig. 4(d)), the area 220 of defrosting of heat exchanger outside the vehicle is further enlargedextended; after the 221 defrosting operates 80 seconds (Fig. 4(e)), the frost layer on the surface of heat exchanger 222 outside the vehicle is basically-melted completely. Only the frost layer on the surface of the 223 flat tube that is close to the bottom doesn't melt; after the defrosting operates 100 seconds 224 (Fig. 4(f)), the defrosting of heat exchanger outside the vehicle complete.

(a)

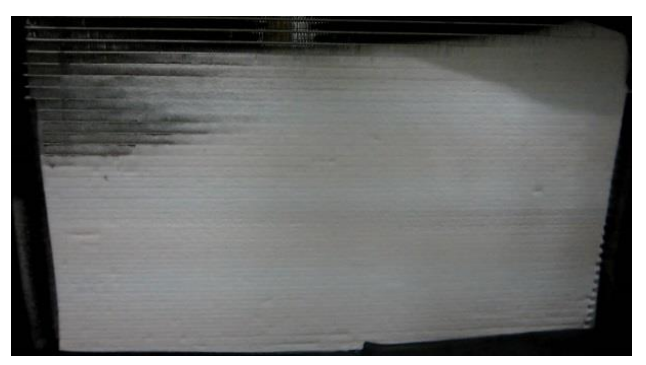

(c) (b)

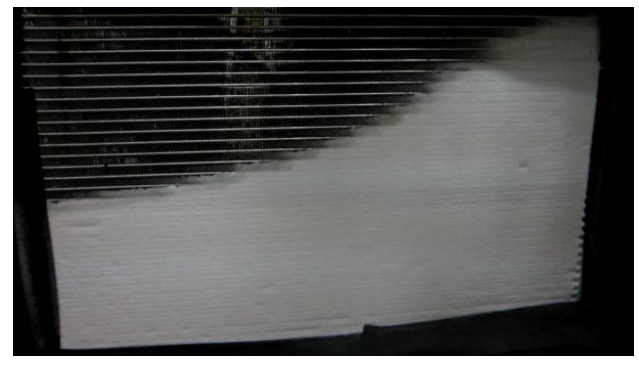

(d) 


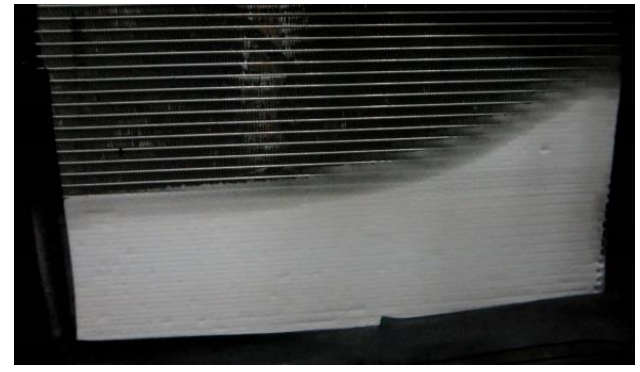

(e)

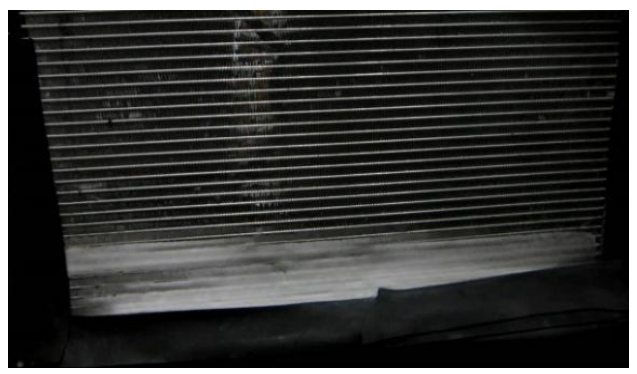

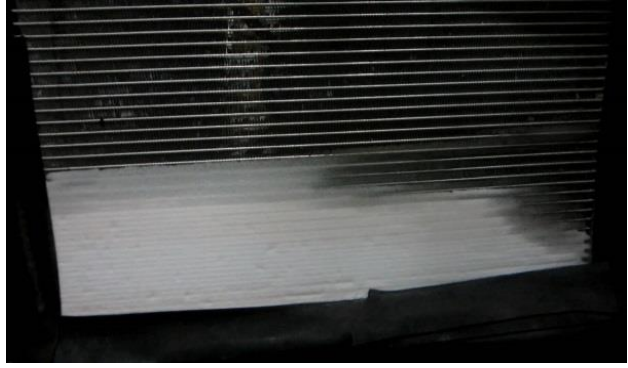

(f)

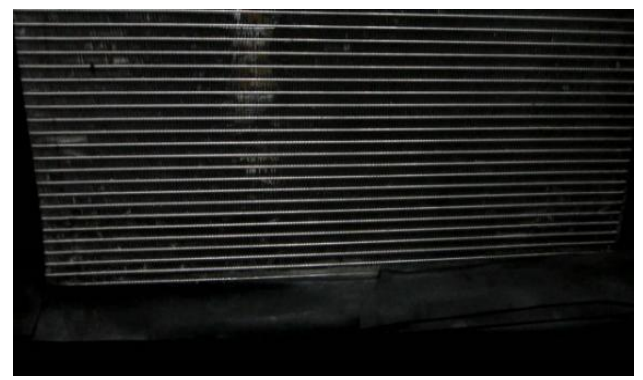

Fig. 4. Defrosting of heat exchanger outside the vehicle: (a) defrosting begins. (b)defrosting operates for 20 seconds.(c) defrosting operates for 40 seconds.(d) defrosting operates for 60 seconds. (e)defrosting operates for 80 seconds.(f) defrosting operates for 100 seconds.

\section{4. Analysis on experiment Experiment results}

\subsection{Influence of environment temperature inside the vehicle on defrosting characteristics}

228 When the ambient temperature outside the vehicle is $0^{\circ} \mathrm{C}$ and the temperatures inside the 229 vehicle are $15^{\circ} \mathrm{C}, 20^{\circ} \mathrm{C}$ and $25^{\circ} \mathrm{C}$ respectively, under frosting conditions, defrost after the 230 frosting completes on the surface of heat exchanger outside the vehicle. When defrosting 231 begins, other parameters shall stay the same with those under frosting conditions. In the 232 defrosting process, the situation that the average condensation temperature and defrosting

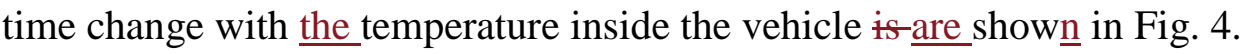

It is shown in Fig. 5 that the higher the environment temperature inside the vehicle, the higher the average condensation temperature in the defrosting process be and the shorter the defrosting time will be. This-It is because when the system is in defrosting mode, the heat exchanger inside the vehicle changes from the condenser to evaporator. The environment inside the vehicle changes from the side of the cold source to the side of the heat source. The

239 higher the environment temperature inside the vehicle, the higher the evaporating temperature 240 will be. The performance of the system will be better. The speed of defrosting accelerates. 


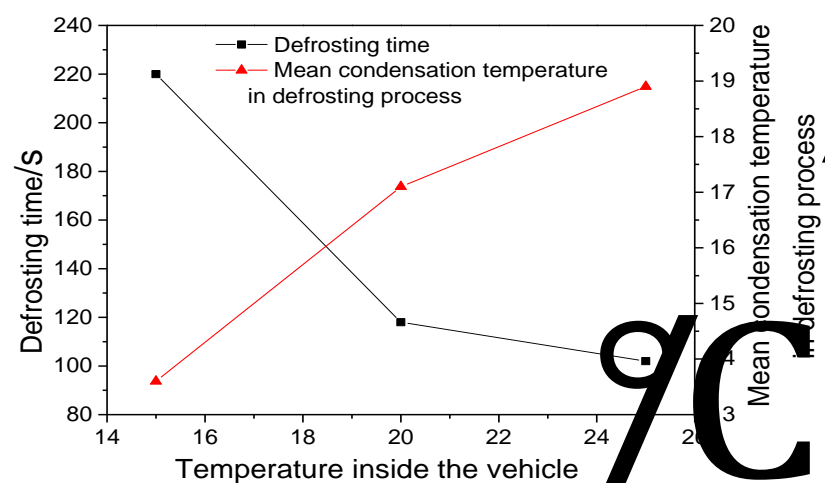

Fig. 5. Influence of temperature inside the vehicle on average condensation temperature and defrosting time during the defrosting process.

\subsection{Influence of revolving speed of compressor on defrosting characteristics}

242

When the ambient temperature outside the vehicle is $0_{-}^{\circ} \mathrm{C}$, and the temperature inside the vehicle is $25_{-}^{\circ} \mathrm{C}$, under frosting conditions, defrost after the frosting completes on the surface of heat exchanger outside the vehicle. When defrosting begins, change the revolving speeds of the compressor into 5000_rpm, 6000_rpm and 7000_rpm respectively and keep other parameters stay the same with those under frosting conditions. The situation that average condensation temperature and defrosting time change with the revolving speed of compressor during the defrosting process is are shown in Fig. 6.

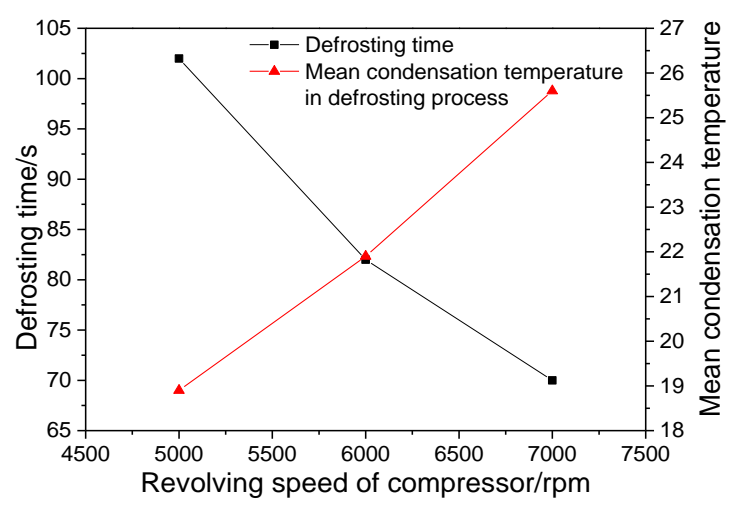

Fig. 6. Influence of revolving speed of compressor on average condensation temperature and defrosting time during defrosting process.

It can be seen from Fig. 6 that the higher the revolving speeds of the compressor, the higher the mean condensation temperature in defrosting process will be and the shorter the defrosting time will be. The revolving speed of compressor increases. The mass flow rate of refrigerating fluid that enters condenser increases. The heat release of condenser increases- ${ }_{2}$ Tthe speedrate of defrosting accelerates [22].

\subsection{Influence of air output of fan inside the vehicle on defrosting characteristics}

When the ambient temperature outside the vehicle is $0_{-}{ }^{\circ} \mathrm{C}$, and the temperature inside the vehicle is $20^{\circ} \mathrm{C}$, under frosting conditions, defrost after the frosting completes on the surface of heat exchanger outside the vehicle. When defrosting begins, change air output of fan inside the vehicle into $450 \_\mathrm{m}^{3} / \mathrm{h}, 500_{-} \mathrm{m}^{3} / \mathrm{h}$ and $540_{-} \mathrm{m}^{3} / \mathrm{h}$ respectively and let other parameters stay the same with those under frosting conditions. The situation that average condensation 
260 temperature and defrosting time change with air output of fan during the defrosting process is 261 are shown in Fig. 7.

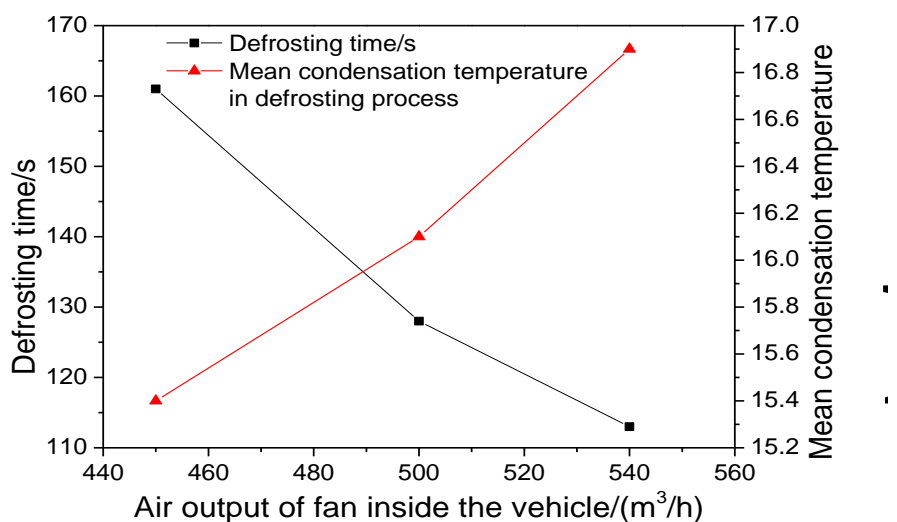

Fig.7. Influence of air output of fan inside the vehicle on average condensation temperature and defrosting time during the defrosting process.

Fig. 7 shows that the greater the air output of fan inside the vehicle, the higher the average condensation temperature in the defrosting process will be and the shorter the defrosting time will be. This-It is because when the system is in defrosting mode, the heat exchanger inside the vehicle changes from the condenser to evaporator. With the increase of air output of fan inside the vehicle, the heat exchange amount of evaporator increases. The heat release of condenser also increases-, Tthe speed-rate of defrosting of heat exchanger outside the vehicle accelerates.

\subsection{Influence of air supplement rate (air recirculation percentage in the air discharge} of compressor) on defrosting characteristics

272 When the ambient temperature outside the vehicle is $0{ }_{-}^{\circ} \mathrm{C}$, and the temperature inside the 273 vehicle is $20{ }^{\circ} \mathrm{C}$, under frosting conditions, defrost after the frosting completes on the surface 274 of heat exchanger outside the vehicle. When defrosting begins, it is necessary to control the 275 air supplement rate at 10\%, 20\% and 30\% and keep other parameters stay the same with those under frosting conditions. The situation that the average condensation temperature and defrosting time during defrosting process change with air supplement rate is shown in Fig. 8.

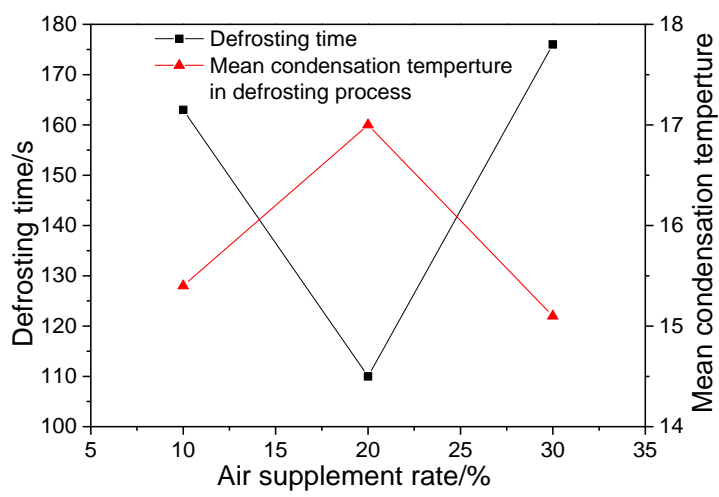

Fig. 8. Influence of air supplement rate on average condensation temperature and defrosting time during defrosting process 
Fig. 8 shows that when the air supplement rate is $20 \%$, the average condensation temperature in the defrosting process is the highest and the defrosting time is the shortest.

280 Compared with the situation that the air supplement rate is $20 \%$, when the air supplement rate is $10 \%$, the mass flow rate of refrigerating fluid in circulation line of air supplement reduces. The heat exchange amount in intermediate heat exchanger between it and working medium of the main line is small It causes that the condensate depression before the main expansion valve is small and leads to the fact that the proportion of working medium of gas state in evaporator increases. The resistance increases. Evaporating temperature and evaporating pressure reduce. It goes against the absorption of the evaporator, thus reduces the heat release of the condenser and prolongs the defrosting time. When the air supplement rate is $30 \%$, although the condensate depression before the main-primary expansion valve increases and the resistance of working medium in evaporator reduces, the mass flow rate of working medium that enters evaporator is small. The influence-impact of it on the reduction of heat absorption capacity of the evaporator is bigger than the influence of reduction of working medium resistance in evaporator on increase of heat absorption capacity of the evaporator. It causes the reduction of total heat absorption capacity of evaporator and heat release of the condenser. Compared with the moment when the air supplement rate is $20 \%$, defrosting time increases.

\subsection{Influence of fan delivery outside the vehicle on defrosting characteristics}

The change of fan delivery outside the vehicle is realized through changing the frequency of fan outside the vehicle. When the ambient temperature outside the vehicle is $0^{\circ} \mathrm{C}$ and the temperature inside the vehicle is $20^{\circ} \mathrm{C}$, under frosting conditions, defrost when the frosting completes on the surface of heat exchanger outside the vehicle. When defrosting begins, change the frequencies of fan outside the vehicle into $0 \mathrm{~Hz}, 5 \mathrm{~Hz}$ and $10 \mathrm{~Hz}$, keep the revolving speed of compressor at 6,000 rpm and keep other parameters stay the same with those under frosting conditions. Figs 9, 10 and 11 show the situations when refrigerating capacity of the system, exhaust gas temperature, outlet air temperature, temperatures at inlet and outlet of heat exchanger outside the vehicle change with time of defrosting operation of the system. With the operation of defrosting, the refrigerating capacity of the system reduces gradually. The exhaust gas temperature reduces firstly and then increases. The outlet air temperature reduces gradually. The temperatures at inlet and outlet of heat exchanger outside the vehicle increases gradually. When the frequency outside the vehicle change with the time of defrosting operation of the system of a fan outside the vehicle is $0 \mathrm{~Hz}$, the refrigerating capacity of the system reduces $26.6 \%$. The outlet air temperature reduces $3.2^{\circ} \mathrm{C}$. When the frequency is $5 \mathrm{~Hz}$, the refrigerating capacity reduces $36.2 \%$. The outlet air temperature reduces $4.9^{\circ} \mathrm{C}$. Although the outlet air temperature reduces, the lowest temperature is above $24^{\circ} \mathrm{C}$. So it will not let people inside the vehicle have the feeling of cold air.

Fig. 12 shows the curve that average condensation temperature and defrosting time change with frequency of fan outside the vehicle in defrosting process. The greater the frequency of

318 fan outside the vehicle, the greater the air output of fan outside the vehicle will be. The 319 average condensation temperature in the defrosting process will be lower. The defrosting time will be longer. This-It is because the system is in defrosting mode. The heat exchanger outside the vehicle serves as the condenser. The air output of fan outside the vehicle increases. 
The condensing temperature of heat exchanger outside the vehicle reduces. The speed of

(a) Fan outside the vehicle $\mathrm{OHz}$

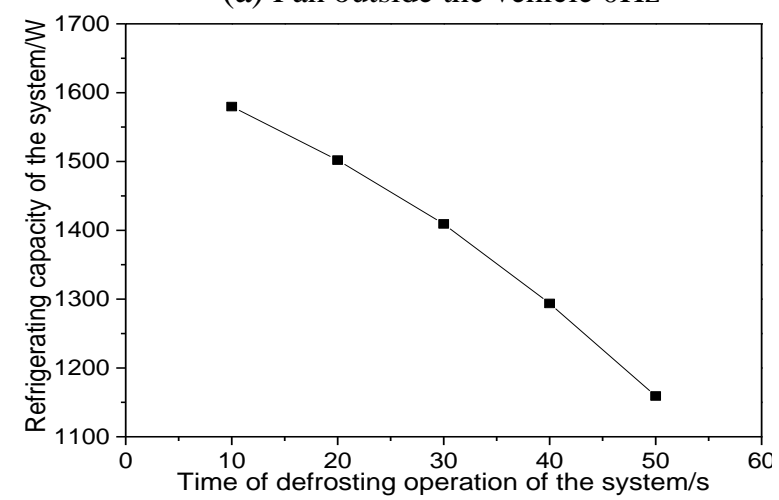

(b) Fan outside the vehicle $5 \mathrm{~Hz}$

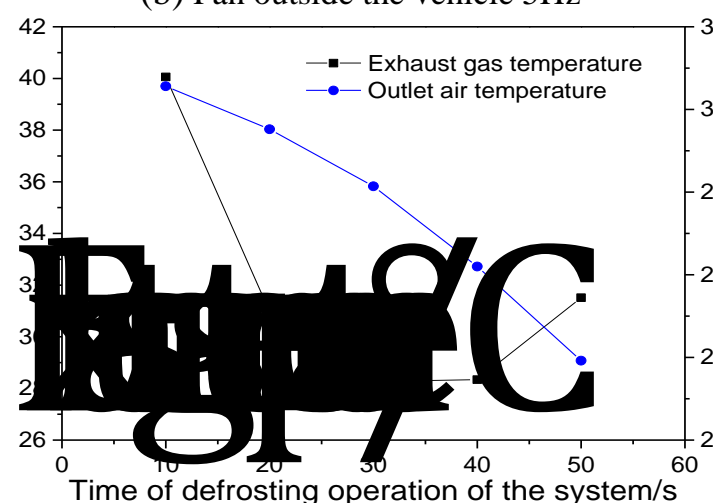

Fig. 9. Curve that refrigerating capacity changes with time of defrosting operation of the system.

(a) )Fan outside the vehicle $0 \mathrm{~Hz}$.

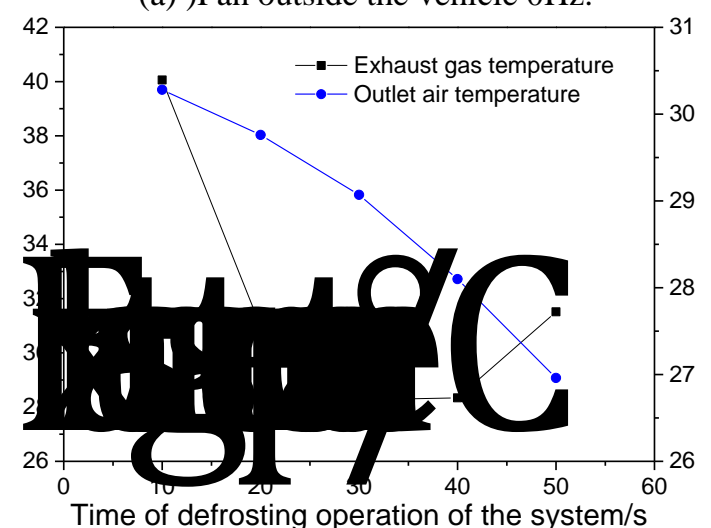

(b) Fan outside the vehicle $5 \mathrm{~Hz}$.

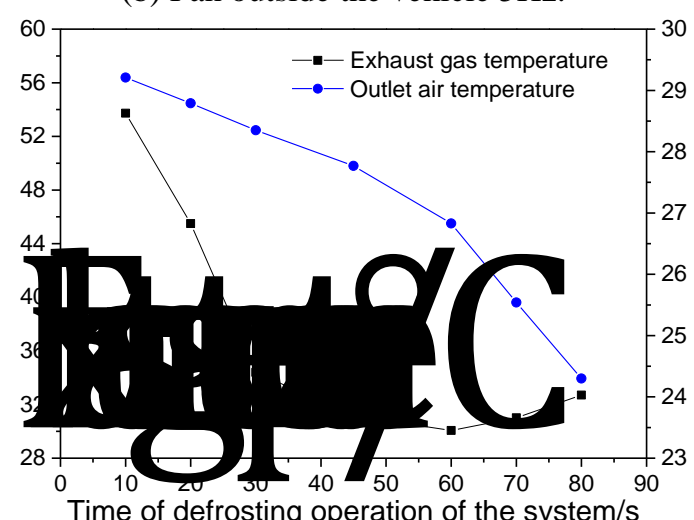

Fig.10. Curve that exhaust gas temperature and outlet air temperature change with time of defrosting operation of the system.

(a) Fan outside the vehicle $0 \mathrm{~Hz}$.

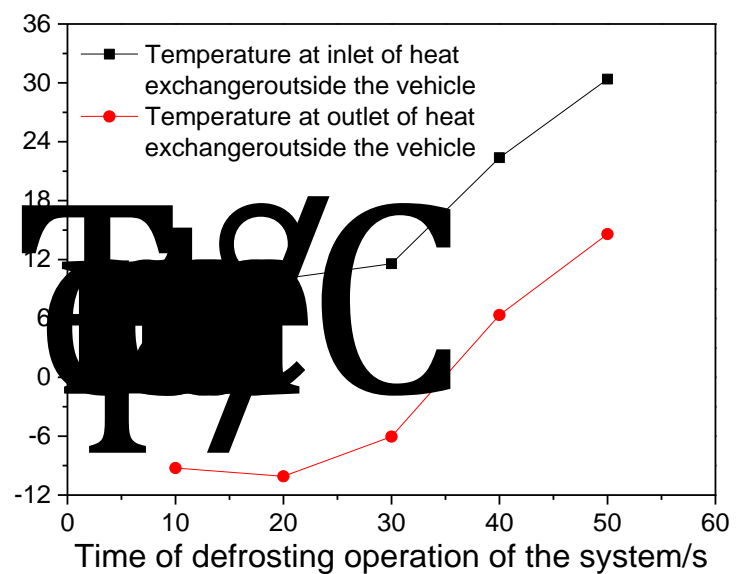

(b) Fan outside the vehicle $5 \mathrm{~Hz}$.

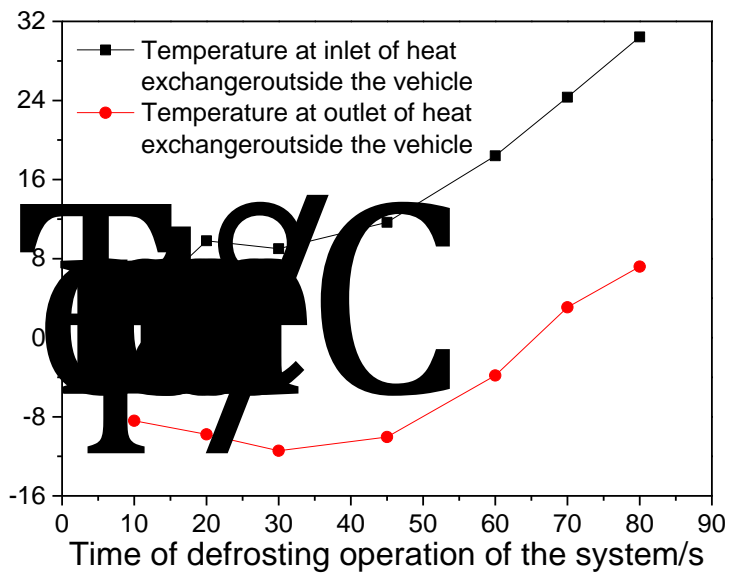

Fig.11. The Ccurve that the temperatures of inlet and outlet of the heat exchanger. 


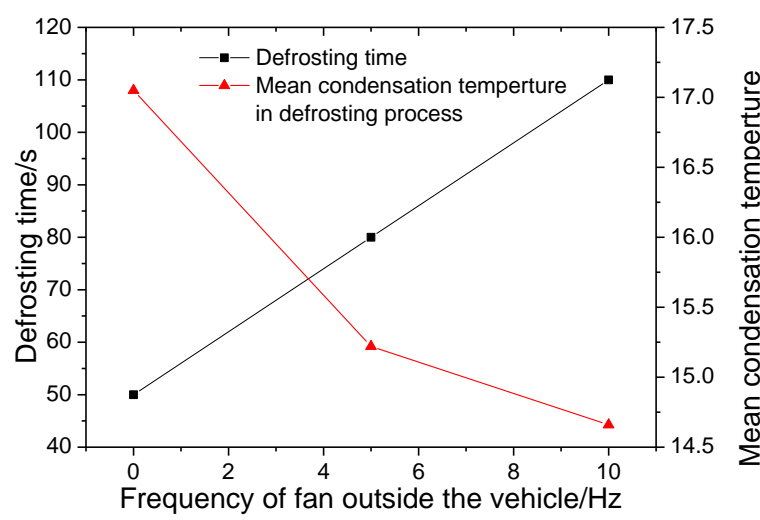

Fig.12. Influence of fan delivery outside the vehicle on average condensation temperature during the defrosting process and defrosting time

\section{Conclusion}

Aiming at the problem that the heat exchanger outside the vehicle is easy to frost when the heat pump type air conditioning system for PEVs operates under the environment of low temperature and high humidity, the article proposes a technical method of fast defrosting relying on increasing temperature and enhancing gas injection in reverse circulation. Following the basic principle of defrosting via reverse circulation, the method well integrates the air speed control outside, air and effect increase of compressor, air intake preheating inside and so on, so that during the defrosting operation of heat exchanger outside, the condensing temperature and heat release in condensing are both increased obviously so as to achieve rapid defrosting. Experimental research shows that it can obviously shorten the defrosting time through raising the in-car temperature, the revolving speed of compressor, air output of draught fan inside the car and reducing fan delivery outside the vehicle and controlling proper air supplement rate, and then adequatelyproperly-improve the performance of air conditioning system to ensure the stable operation of air conditioning system of PEVs.

\section{Acknowledgement}

342 This work was supported by the Research Funds of Key Laboratory of Cryogenics, TIPC, 343 CAS (No. CRYO201509).

\section{References}

1. ZhanFeng Liu,Li Song,DanPing Zhao,et al.Automotive air conditioning.Peking University

2. Fei Qin,QingFeng Xue,Giovannyy Marcelo Albarracin Velez,et al.Experimental Investigation on Heating Performance of Heat Pump for Electric Vehicles at $-20{ }^{\circ} \mathrm{C}$ Ambient Temperature,Energy Conversion and Management102(2015)39-49.

3. HuiMing Zou,Wei Wang,GuiYing Zhang,et al.Experimental Investigation on an Integrated Thermal Management System with Heat Pipe Heat Exchanger for Electric Vehicle,Energy Conversion and Management118(2016)88-95. 
4. FuLong Yan,Design and Performance Simulation of Heat Pump Air Conditioning System for Pure

Electric Passenger Car, JiLin University,2012[in Chinese].

5. Zhaogang Qi, Jiangping Chen, Zhijiu Chen,et al.Experimental study of an auto-comtrolled automobile air conditioning system with an externally-controlled variatle displacement compressor,Applied Thermal Engineering27 (2007)927-933.

6. Changqing Tian, Xianting Li,Numerical simulation on performance band of automotive air conditioning system with a variable displacement compressor,Energy Conversion and Management,46(2005)2718-2738.

7. NengZhao Jiang, Heat pump technology and applications for air conditioning. Mechanical Industry Press, 1997[in Chinese].

8. Zhe Zhang, JinJin Tian, Experiment Research under Working Condition of Frosting of Air Cooled Heat Pump, Refrigeration and Air-conditioning22 (1)(2008) 88-90.

9. Haijun Li,GuangHui Zhou, AnGui Li, et al. Research on Frost Characteristics of Heat Pump Type Air Conditioning System for Pure Electric Vehicles, Cryogenics and Superconductivity42(9)(2014) 6063[in Chinese].

10. Sanders C T. Frost formation: The influence of frost formation and defrosting on the performance of air coolers[D]. Netherlands: Delft University, 1974.

11. АНДРАЧНИКОВ Е N, et al. Ву JinDuo Yang translated into Chinese. The efficient way of evaporator defrost automatically, Refrigeration 03 (1986)74-78.

12. Payne V, O'Neal D L. Effects of Outdoor Fan Airflow on the Frost/Defrost Performance of An AirSource Heat Pump, Texas,United States: American Society ofMechanical Engineers, Advanced Energy Systems Division (Publication) AES, 1993.

13. Padhmanabhan S, Cremaschi L, Fisher D E, et al. Comparison of Frost and Defrost Performance Between Microchannel Coil and Fin-and-Tube Coil for Heat Pump Systems, International Refrigeration and Air Conditioning Conference, Purdue,USA, 2008.

14. Z Liu, G Tang, F Zhao,Dynamic Simulation of Air-Source Heat Pump During Hot-gas Defrost, Applied Thermal Engineering 23(6)(2003)675-685.

15. Dopazo J A, Fernandez-Seara J, Uhía F J, et al. Modelling and Experimental Validation of the Hot-Gas Defrost Process of an Air-Cooled Evaporator, International Journal of Refrigeration33(4) (2010)829839.

16. M Qu, D Pan, L Xia, et al. A Study of the Reverse Cycle Defrosting Performance on a Multi-Circuit Outdoor Coil Unit in an Air Source Heat Pump - Part I:Experiments ,Applied Energy91(1) (2012) 122129.

17. M Qu, D Pan, L Xia, et al. A Study of the Reverse Cycle Defrosting Performance on a Multi-Circuit Outdoor Coil Unit in an Air Source Heat Pump - Part II:Modeling Analysis, Applied Energy91(1) (2012)274-280.

18. Hua Zhong,ShuangBo Tang,ZhiJiu Chen,et al.Research on Defrosting Experiment of Air Conditioning Evaporator in Cars, Fluid Machinery29(1)(2001) 44-46[in Chinese].

19. Jianghong Wu, Fang Xie, ChaoPeng Liu, et al.Research on Adaptation of Heat Exchanger of Microchannel in Heat Pump Air Conditioning System of Electric Vehicles, Journal of Mechanical Engineering 48(14)(2012) 141-147[in Chinese].

20. BaoLong Wang, Application Research on the Scroll Compressor with Refrigerant Injection, Thinghua University,2006[in Chinese].

21. HaiJun LI, GuangHui ZHOU, AnGui LI, et al. Heat Pump Air Conditioning System for Pure Electric Vehicle at Ultra Low Temperature, Thermal Science5(2014) 224-229.

22. Xiao Yang, YuYing Yan,Molecular Dyanmics Simulation for Microscope Insight of Water Evaporation on a Heated Magnesium Surface,Applied Thermal Engineering 31(5)(2011)640-648 